\title{
Surface Embedding, Topology and Dualization for Spin Networks.
}

\author{
Peter Kramer ${ }^{a}$ and Miguel Lorente ${ }^{b}$, \\ ${ }^{a}$ Institut für Theoretische Physik der Universität D 72076 Tübingen, Germany, \\ ${ }^{b}$ Departamento de Fisica, Universidad de Oviedo, E 33007 Oviedo, Spain.
}

May 15, 2002

\begin{abstract}
.
Spin networks are graphs derived from $3 n j$ symbols of angular momentum. The surface embedding, the topology and dualization of these networks are considered. Embeddings into compact surfaces include the orientable sphere $S^{2}$ and the torus $T$, and the not orientable projective space $P^{2}$ and Klein's bottle $K$. Two families of $3 n j$ graphs admit embeddings of minimal genus into $S^{2}$ and $P^{2}$. Their dual 2-skeletons are shown to be triangulations of these surfaces.
\end{abstract}

\section{Spin networks.}

Spin networks were introduced by Penrose [13] as a purely combinatorial description of the geometry of spacetime. A spin network has a graph $\Gamma$ of $3 n$ edges which represents an invariant formed from $3 n$ angular momenta $j$. $\Gamma$ is connected and at any vertex has degree 3 . When $j$-values are attached to $\Gamma=\Gamma(3 n j)$ which fulfill the intertwining rules at each vertex, the value of the corresponding $3 n j$ invariant can be computed and used in the theory of spin networks. A systematic description and evaluation of the $3 n j$ invariants is given by Yutsis et al. in [19]. Motivated by the discretization of a Riemannian surface to avoid infinities in Einstein action, Ponzano and Regge 14] extensively used $3 n j$ symbols and their geometrical interpretation. Hasslacher and Perry [6] proved that Ponzano Regge discretization is a particular case of spin networks. The development of this subject is reviewed in [16]. The spin network approach uses combinatorial properties of a set of relations to give an interpretation of the structure of space. In the present paper we explore a similar correspondence between combinatorial properties of graphs and their embedding in 
compact surfaces. Our method is in line with algebraic topology and provides a more rigorous setting for the intuitive embedding of $3 n j$ symbols given by Ponzano and Regge. The philosophical background to a spin network interpretation goes back to the relational theory of space and time proposed by Leibniz. One of the authors (M.L.) has developed in [10, [11] a fundamental theory based on Leibniz' ideas to give an interpretation of discrete models of space and time.

\section{Topology of spin networks.}

We turn to the topological aspects of spin networks. Underlying a spin network is a graph $\Gamma$. If its vertices are taken as points and its edges as lines, its geometry provides a 1-skeleton. We shall use this and other notions of cell complexes taken from algebraic topology [12. A topological analysis of spin networks in our opinion would set the ground for the following explorations:

(1) Ponzano and Regge in their work 14] of 1968 discuss extensively graphs for $3 n j$ symbols and speak about surfaces, polytopes and subdivisions associated with them. In their approach to discrete quantum gravity they consider the $3 n j$ symbols, starting from $6 j$, as polytopes in $E^{3}$. Topological properties of higher symbols are sketched only for the $9 j$ symbol. But many of the general spin networks with $3 n j$ graphs, beginning with $\Gamma(9 j)$ as given by Yutsis [19] p. 60 Fig. 18.1, do not admit an orientable embedding into $S^{2}$ and $E^{3}$. It will be necessary to restrict the class of spin networks or else to find new ways of dealing with their embedding. Recent mathematical analysis can provide and classify the topology of $3 n j$ graphs and their variety.

(2) In the discussion of spin networks there appears the notion of duality, compare for example Baez [1. Since the graphs of spin networks have degree 3 at any vertex, it is conjectured that the dual has triangular faces. This triangulation is also anticipated as a geometrical tool in [14. But duality of networks, in the notion of algebraic topology [12, works only provided the graph $\Gamma$ has been embedded into a surface. So embedding must precede dualization and triangulation.

(3) A graph $\Gamma$ seen from its vertex (point) set $V$ is a combinatorial rule to select pairs of vertices linked by a set of edges $E$. A natural extension from the notion of a graph to a richer geometry and topology is to consider closed sets of edges on $\Gamma$ as candidates for faces. The analysis of surface embeddings allows to equip families of graphs with faces and topological data like Euler characteristics, orientation and genus obtained in this process. The universal covering of the embedding surface provides a corresponding covering of the embedded graph. Models for physics with discrete geometry could take advantage of this access. Regge [15] has shown how geometric notions such as curvature arise in discrete geometries. 
In what follows we restrict the analysis of spin networks to topological questions. We analyze for graphs the embedding, topology (Euler characteristics $\chi$ and genus $g$ ), dualization and possible triangulation under the following restrictions:

(i) The graphs $\Gamma$ will be taken from spin networks. The corresponding restrictions on the graphs as given in [19] are summarized in section 6 .

(ii) We introduce faces into the graphs of spin networks, but restrict their choice such that all of them can be embedded into a single compact surface $\mathcal{S}$.

The dimension $d=2$ is sufficient for studying duality and may be taken towards $d>2$ in later steps, see section 6 . Compactness excludes in particular embeddings into manifolds with boundaries. So closure is in line with the standard notions of space in physics. The compact surfaces admit a well-known classification of their topology [18] which we briefly review in section 3. They are uniquely characterized by their Euler characteristics, orientability and genus. Embeddings restricted to these surfaces become accessible to mathematical analysis. In section 4 we describe the theory for the embedding of graphs into surfaces. This theory was given in 1995 by Bonnington and Little [3], it was reanalyzed and presented as an algorithm by Schwark [17. In section 5 we apply the method to the simplex. We demonstrate the variety of surfaces including $S^{2}, T, P^{2}$ and Klein's bottle $K$, and genus ranging as $g=0,1,2,3$. In section 6 we find embeddings of minimal genus for two families of $3 n j$ graphs. In section 7 we dualize the 2-skeleton for the minimal embeddings and find triangulations of the surfaces $S^{2}, P^{2}$. In the section 8 conclusion we comment on implications of topological properties for spin networks.

\section{Compact surfaces and topological polygons.}

A topological surface $\mathcal{S}$ we take as a compact 2-manifold. This means that we exclude manifolds with boundaries like the cylinder or the Möbius strip. For elementary concepts and illustrations in the topology of surfaces we refer to Hilbert and CohnVossen [7], sections 44-51, pp. 290-340.

The topology of compact surfaces can be described by means of systems of topological polygons, see Seifert and Threlfall [18]: Consider in $E^{2}$ a system $\mathcal{M}$ of $F$ polygons with edges and vertices, each homeomorphic to a unit disc, with pairwise disjunct point sets. Assume that the number of edges is even and that there is a topological map of each edge and its vertices onto exactly one other edge and its vertices. Identify pairwise the edges and their vertices under this map. Then the system $\mathcal{M}$ is called a topological polygon.

Each polygon of $\mathcal{M}$ can be given a coherent orientation of its edges [18] p. 132, 12 pp. 26-33. First one chooses an individual orientation on each edge, consistent with the topological identification. Then one chooses a single orientation on any consecutive sequence of edges forming a polygon. The orientation of the polygon induces an orientation on the edges belonging to it. In relation to the individual 
orientation it is marked by an exponent \pm 1 .

If the orientations of all polygons of $\mathcal{M}$ can be chosen such that any edge gets two opposite induced orientations, $\mathcal{M}$ is called orientable, otherwise nonorientable.

By $|V|,|E|,|F|$ denote the number of vertices, edges and faces of a polygon . Then the Euler characteristics $\chi$ is defined by

$$
\chi=|V|-|E|+|F|
$$

A topological polygon can be simplified into a fundamental polygon without change of the orientability and characteristics. These properties uniquely classify the compact surfaces. The genus $g$ of the surface $\mathcal{S}$ is determined by the Euler characteristics and the orientability:

$$
\begin{aligned}
\mathcal{S} \text { orientable } & : \quad \chi(\mathcal{S})=2-2 g(\mathcal{S}), \\
\mathcal{S} \text { nonorientable } & : \quad \chi(\mathcal{S})=2-g(\mathcal{S}) .
\end{aligned}
$$

In Table 1 we give from [18 the topological data of the sphere $S^{2}$, the projective space $P^{2}$, the torus $T$, and Klein's bottle $K$. The projective sphere can be described by closing the boundary of a Möbius strip with a cross cap. In Figs. 1,2 we sketch the fundamental polygons of these four surfaces. The non-orientable surface $C^{2}$ with $\chi=-1, g=3$ will appear in section 5 as an embedding of the simplex. $C^{2}$ has three cross-caps.

Table 1: Euler characteristics $\chi$ and genus $g$ of fundamental polygons for the sphere $S^{2}$, the projective space $P^{2}$, the torus $T$, Klein's bottle $K$, and a non-orientable surface $C$.

\begin{tabular}{llllll}
\hline Name : & $S^{2}$ & $P^{2}$ & $T$ & $K$ & $C^{2}$ \\
\hline$|V|$ & 2 & 1 & 1 & 1 & 1 \\
$|E|$ & 1 & 1 & 2 & 2 & 3 \\
$|F|$ & 1 & 1 & 1 & 1 & 1 \\
$\chi$ & 2 & 1 & 0 & 0 & -1 \\
Genus g & 0 & 1 & 1 & 2 & 3 \\
Orientable & yes & no & yes & no & no \\
\hline
\end{tabular}

In Figs. 1 and 2 we sketch the fundamental polygons for the first four surfaces of Table 1. 

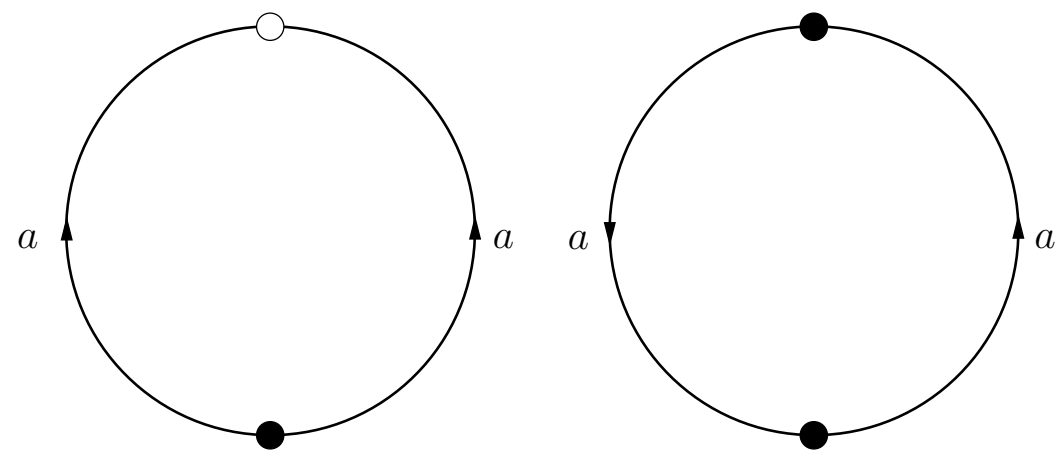

Fig. 1. Fundamental polygons for the sphere $S^{2}$ with two vertices, one edge $a$ and one face (left), and for the projective space $P^{2}$ with one vertex, one edge $a$ and one face (right). The arrows determine the gluing of edges.
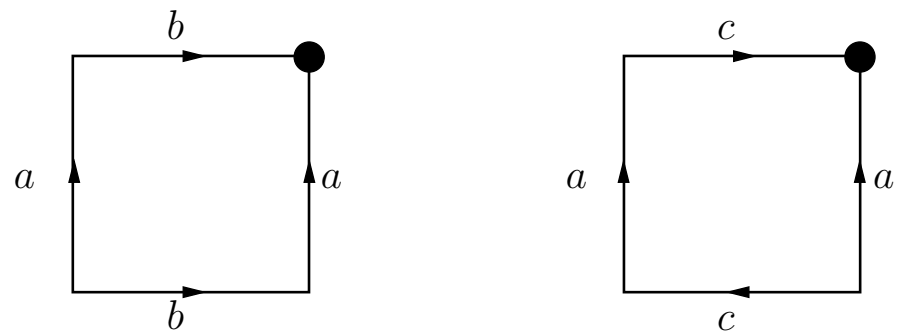

Fig. 2. Fundamental polygons for the torus $T$ with one vertex, two edges $a, b$ and one face (left), and Klein's bottle $K$ with one vertex, two edges $a, c$ and one face (right).

We shall use in particular the sphere $S^{2}$ and the projective space $P^{2}$ for the embeddings.

In Figs. 4 and 5 we follow Seifert and Threlfall [18 p. 10 and present the nonorientable surface $P^{2}$ and its tesselations as a sphere: The upper und lower hemisphere are projected to a single equatorial plane containing the equator, and opposite points on the equator are identified.

\section{Embedding of a graph into a surface.}

For notions of graph theory we refer to Biggs [2]. A graph $\Gamma$ consists of two sets $V \Gamma, E \Gamma$, called the vertices and edges of $\Gamma$ with elements $v, e$ respectively, and an incidence relation, that is, a subset of $V \Gamma \times E \Gamma$. We require that every edge is incident with two vertices, and no two edges are incident with the same pair of vertices, that is, the graph $\Gamma$ has no loops. We follow the exposition of Schwark [17] of results due to Bonnington and Little [3]. 
We define an embedding of a graph $\Gamma$ into a surface. Let $\Gamma$ be a graph and $\mathcal{S}$ be a compact 2-manifold or surface $\mathcal{S}$. A drawing of $\Gamma$ on $\mathcal{S}$ is a map of the vertices and edges into points and curves on $\mathcal{S}$ which preserves the relation between edges and vertices and moreover has no intersection of edge curves except in their common vertices. Define the components of $\mathcal{S}$ after removal of all edge curves as the regions of the drawing. If all the regions of the drawing are homeomorphic to the Euclidean plane $E^{2}$ we call the drawing an embedding of $\Gamma$ into $\mathcal{S}$.

Given such an embedding of a graph, we may attach the Euler characteristics and the genus $\chi, g$ to the embedded graph. Given a graph, its embedding into a compact surface is not unique. We speak of a minimal embedding if the genus $g$ of the embedding surface is minimal.

We now follow [17] and describe the steps which lead to embeddings of a given graph into surfaces. A graph $\Gamma$ can be reconstructed by incidences from its set of edges as follows. Start from a set $E,|E|$ and define on $E X=: E \times\langle-1,1\rangle,|X|=2|E|$ with elements $x \in X$. The edge set $E=E(X)$ in terms of $X$ is defined as the set of (unordered) pairs $e:=((e,-1),(e, 1)) \in X \times X$. The pair $((e,-1),(e, 1))$ forms the negative and positive ends [2] p. 24 of the edge $e$. Choose the set $V(X)$ as a partition $V$ of $X$ into subsets $v_{\alpha}, v_{\beta}, \ldots$ such that the subset $v_{\beta}$ reproduces the incidences of the ends of edges at the vertex $v_{\beta} \in V(\Gamma) \subset \Gamma$. It follows that from $(X, E, V)$ we can reconstruct $\Gamma$.

Now choose two bijections $\pi, \phi$ of $X$ as follows: The orbits on $X$ under $\pi$ are the sets of ends for any vertex. Therefore $\pi$ is a unique involutive bijection which interchanges the negative and positive ends on all the edges. The bijection $\phi$ is chosen such that the orbits on $X$ under $\phi$ are all the vertex subsets $v_{\beta} \in X$. The choice of $\phi$ is not unique and becomes part of the classification of embeddings of $\Gamma$. Given $(X, E, V)$, any possible choice of $\phi$ provides an order among the edges incident with any fixed vertex. Define a map $\mathcal{M}$ as a triple $((X, E, V), \pi, \phi)$ with a fixed choice of $\phi$.

From a map $\mathcal{M}=((X, E, V), \pi, \phi)$ with underlying graph $\Gamma$, we now assign a signature $i$ to its edges. We form $X \times\langle-1,1\rangle$ with elements $(x,-1),(x,+1)$. The signature of an edge is a function $i(e)= \pm 1$. For $(x, \pm 1) \in e$ we also assign $i((x, \pm 1))=i(e(x))=i(e)$. A map whose edges are equipped with a signature is denoted as $\mathcal{M}^{i}$. For any element $(x, k) \in X \times\langle-1,1\rangle$ and given signature we define [17] an operator

$$
\Pi:(x, k) \rightarrow \Pi(x, k)=\left(\phi^{k i(e(x))} \pi x, k i(e(x))\right)
$$

$\Pi$ can be shown [17] to be a bijection of $X \times\langle-1,1\rangle$. Moreover its orbits by construction pick one and only one point from each edge. These orbits therefore define a sequence of consecutive edges from the graph $\Gamma$. Each orbit of edges under $\Pi$ determines a face of an embedding of the map $\mathcal{M}^{i}$ into a surface. If there are $|F|$ orbits, the Euler characteristics of this surface is obtained by application of eq. 11. The orientability of an embedding can be decided as described in section 3 . The sets 
of consecutive edges become the orbits of edges from the map eq. 3. Then eq. 2] can be used to find the genus $g$.

The topology of a surface $\mathcal{S}$ is further characterized by their homotopy and homology groups. These topological data must also characterize the embedded cell complexes. We refer to [18] for details on these groups.

\section{Simplex embedding into compact surfaces.}

We demonstrate the algorithm of Schwark [17 on the graph $\Gamma$ for a simplex. The simplex corresponds to the $6 j$ symbol. It is the first non-trivial spin network and is used in the geometric approach to quantum gravity due to Ponzano and Regge [14. A planar graph $\Gamma$ for the simplex is shown in the last entry of Fig. 3. We label the edges by the letters $a, b, c, d, e, f$. To each edge we attach first an initial and a final point $x$. These points fall into 4 vertex sets. To each point we assign $k= \pm 1$. This gives the set $X \times\langle 1,-1\rangle$ of 24 signed points $(x, s)$. The bijection $\pi$ interchanges the initial and final point on any vertex without changing the sign. The bijection $\phi$ must have the vertex sets of points as its orbits. Therefore for the simplex, and more generally for any graph of degree 3 at any vertex, $\phi$ is the direct product $(Z / 3 Z)^{4}$ of 4 cyclic groups of order 3 at the four vertices. Our first choice $\phi=\phi_{1}$ for the simplex is the product of counterclockwise cycles at all 4 vertices. Next we choose the signature $i= \pm 1$ on the edges. We consider after one another signatures with $0, \ldots, 6$ negative signs. For choosen signature we start the Schwark algorithm and determine the orbits on $X \times\langle 1,-1\rangle$ under the bijection $\Pi$. Our choices of signatures are given in Table 2 for each running number $m$ in alphabetic order $(a, b, c, d, e, f)$ of the edges. Each orbit of points determines an orbit of edges, but any edge appears twice in different orientation. In Fig. 10 we draw the orbits of edges as continuous lines running parallel to a sequence of edges of the graph $\Gamma$. The number of edge orbits gives the number $|F|$ of faces of the embedding. Together with $|V|=4,|E|=6$ this determines the Euler characteristics $\chi$ eq. 1 and from eq. 2 the genus $g$. All results for our choice of $\phi$ are summarized in Table 2. Observe that any edge of $\Gamma$ appears exactly twice in the orbits. This property follows from the assumed closure property of the embeddings which excludes edge lines containing points on a boundary.

To check the orientability we choose the individual orientations on the 6 edges as shown by white arrows in the last entry of Fig. 3. The induced orientations on orbits of edges are then marked by exponents \pm 1 as given in Table 2. In Fig. 3 a chosen orientation on polygon faces is marked by black arrows. All induced orientations on faces are marked by their exponents in Table 2 . 

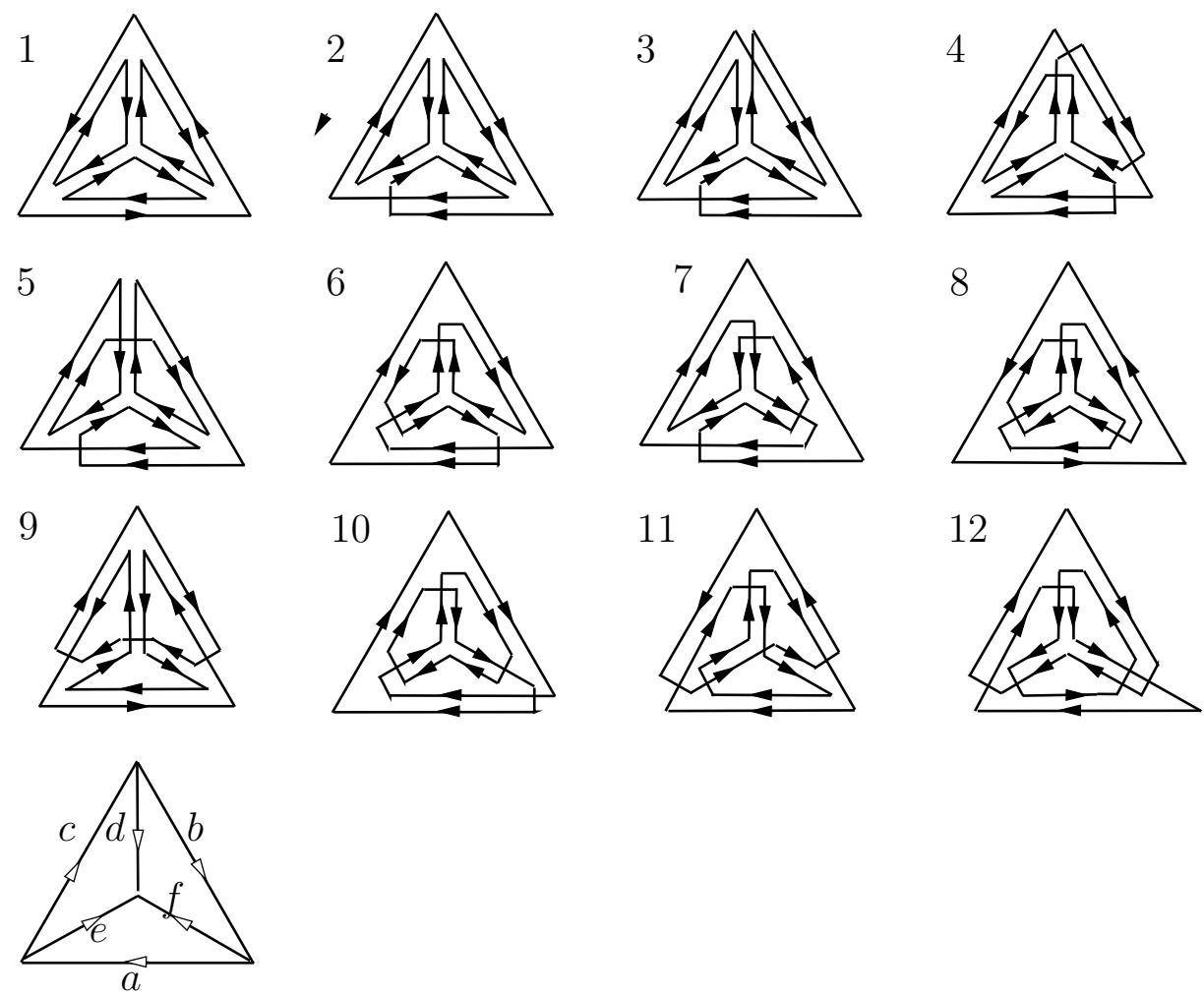

Fig. 3. 12 embeddings of the simplex graph $\Gamma$ into compact surfaces. The last drawing gives the notation for edges of $\Gamma$ and their individual orientation by white arrows. Any face of the 12 embeddings is drawn as a continuous line running piecewise parallel to an orbit of edges of $\Gamma$ and oriented by the black arrows. Any edge appears twice on these lines (closure!). Crossings of lines do not imply intersections. The topological data, the orbits of edges which form faces, and the induced orientations are listed in Table 2.

Table 2: 12 embeddings of the simplex into compact surfaces. The columns give for $m=1, \ldots, 12$ the signature $i$, the number of faces $|F|$, the characteristics $\chi$, the genus $g$, a symbol for $\mathcal{S}$ and its orientability $y / n o$, and orbits of edges for faces with exponents for their induced orientation. 


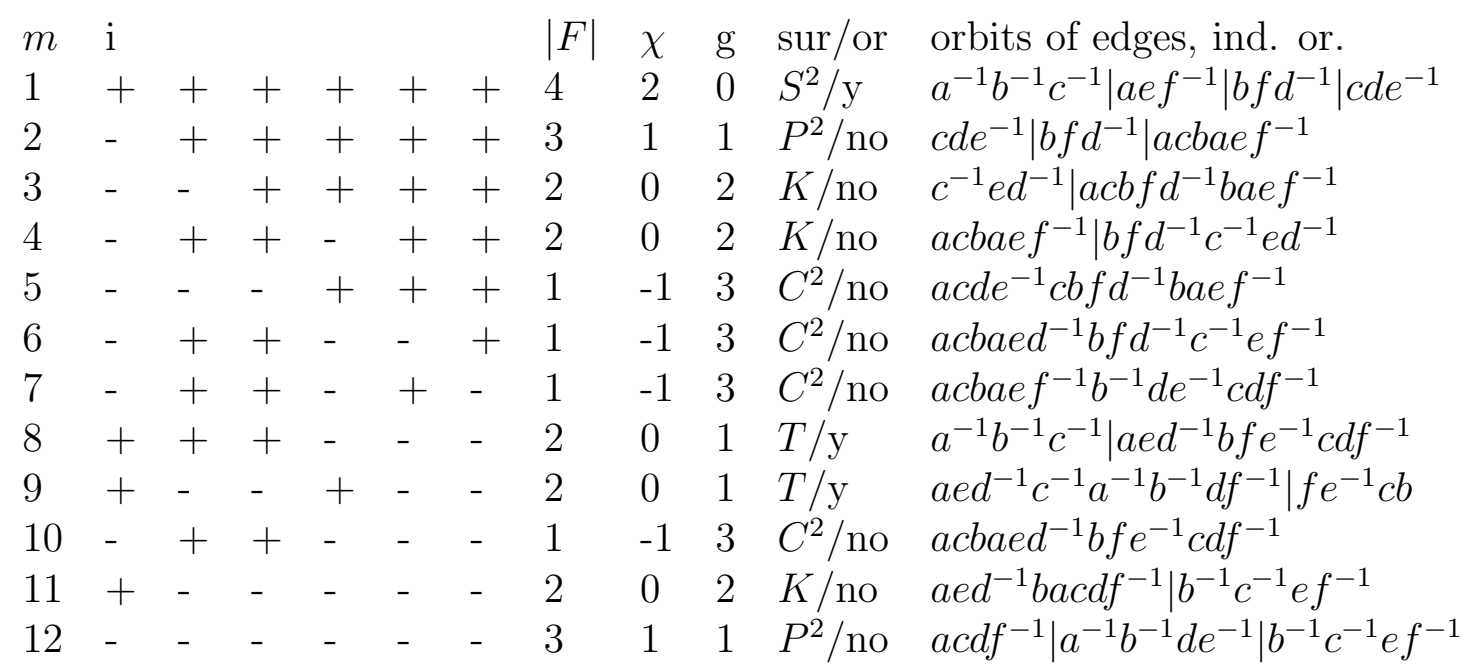

The orientability with the criteria given in section 3 can be easily decided by inspection of the drawings in Fig. 3: Only in the drawings with numbers $(1,8,9)$ is it possible to give each edge two opposite induced orientations. In all other cases, allowing for the inversion of the orientation on faces, no induced orientation can match all the pairs of edges with opposite arrows. The topological polygons for $S^{2}, P^{2}, T, K$ are given in section 3. They would result from the embedded graphs by applying the topological reduction algorithm given in [18] pp. 135-140. Table 2 does not exhaust the simplex embeddings since we made only one choice of $\phi$. A straightforward but lengthy computation would yield all possible embeddings.

There is a single embedding (1) of minimal genus $g=0$ into the sphere $S^{2}$, and there are two more orientable embeddings $(8,9)$ into the torus $T$. Two non-orientable embeddings $(2,12)$ go into $P^{2}$, three $(3,4,11)$ into $\mathrm{K}$. The four non-orientable embeddings $(5,6,7,10)$ have $\mathcal{S}=C^{2}$ of genus $g=3$. $C^{2}$ has 3 cross-caps, and there is a mirror pair $(6,7)$.

We now consider the dualization $\Gamma \rightarrow \Gamma^{*}$, compare section 7 , of the simplex $\Gamma \subset \mathcal{S}$ in the sense of algebraic topology [12. It depends on the chosen embedding. Under dualization of a 2-skeleton, vertices are interchanged with faces, and edges go into edges. The topological data $\chi, g$ of the embedding in each case stay the same.

The numbers of duals are

$$
\left|V^{*}\right|=|F|,\left|E^{*}\right|=|E|,\left|F^{*}\right|=|V|
$$

and for the simplex from Table 2 range as $\left|V^{*}\right|=4,3,2,1,\left|E^{*}\right|=6,\left|F^{*}\right|=4$. All the cell complexes dual to the simplex are distinct triangulations of their compact surfaces and obey the rule $2\left|E^{*}\right|=3\left|F^{*}\right|$ typical for these. For the embedding (1) of minimal genus $g=0$ into $S^{2}$, the dual $\Gamma^{*}$ is the second simplex often considered in relation with the $6 j$ symbol. 


\section{Graphs $\Gamma(3 n j)$ embedded into compact surfaces.}

The graphs $\Gamma=\Gamma(3 n j)$ are characterized by the properties [19]:

( $\Gamma 1)$ : Each graph $\Gamma$ is connected and has $|E|=3 n$ edges,

$(\Gamma 2)$ : The local degree at any vertex is three,

(Г3): The graph $\Gamma$ cannot be separated into two disjoint parts by cutting less than four lines.

Yutsis et al. [19] describe in particular $3 n j$ symbols and graphs of a first and a second type. Moreover they give on 19 pp. 65-70 methods by which new $3 n j$ invariants $\Gamma^{\prime}$ can be recursively constructed: They are generated from a known $\Gamma$ by putting two additional vertices on any two lines and connecting them. From the Schwark algorithm we must expect for these graphs $\Gamma^{\prime}$ embeddings into surfaces $\mathcal{S}$ of increasing genus $g$.

In what follows we shall consider only the first and second type of $3 n j$ symbols and for short denote them by $3 n j(1), 3 n j(2)$. In [19] one finds planar graphs for these symbols. The graphs for symbols of the second type can be redrawn in the plane without crossing of edges. The planar graphs for symbols of the first type differ from the ones of the second type by a twist of two edges. Therefore they cannot be drawn in the plane without a crossing of edges. We recall: A graph $\Gamma$ which cannot be drawn on the plane without crossing of edges cannot be embedded into $S^{2}$ without crossing of edges. If a graph can be embedded into $S^{2}$ without crossing, it is topologically equivalent to a convex polytope. But any convex polytope can be represented by a Schlegel diagram on the plane without crossing. It follows that the $3 n j$ graphs of the first type cannot be embedded into the sphere and has minimal embedding into $P^{2}$. The analysis of the simplex given in section 6 shows that there will in general be a wide variety of embeddings. From Yutsis [19] we know that the number of $n j$-symbols with different graphs will increase with $n$. The examples of the simplex $6 j$ and of the $9 j$ symbol show that we must expect embeddings into compact surfaces of increasing genus $g$.

Our results on $3 n j$ graphs is obtained by a direct construction:

1 Prop: The $\Gamma(3 n j)$ graphs of the first type admit polygonal embedding into the non-orientable projective space $P^{2}$ of minimal genus $g=1$. The minimal property results from the remarks made above. The topological data are listed in Table 3. Examples are given in Figs. 4 and 5 for the $9 j(1)$ and $12 j(1)$ symbols. Recall that objects in opposite position must be identified.

The embedding of the $9 j(1)$ graph into $P^{2}$ was already given by Ponzano and Regge 14.

2 Prop: The $\Gamma(3 n j)$ graphs of the second type admit a polygonal embedding into the orientable sphere $S^{2}$ of minimal genus 0 . The topological data are listed in Table 4. The example of $12 j(2)$ is shown in Fig. 6.

Table 3: Polygonal embedding for $\Gamma(3 n j)$ graphs of first type. 


\begin{tabular}{lllll}
\hline Name & $9 j$ & $12 j$ & $15 j$ & $3 n j$ \\
\hline$|V|=\left|F^{*}\right|$ & 6 & 8 & 10 & $2 n$ \\
$|E|=\left|E^{*}\right|$ & 9 & 12 & 15 & $3 n$ \\
$|F|=\left|V^{*}\right|$ & 4 & 5 & 6 & $n+1$ \\
$\chi$ & 1 & 1 & 1 & 1 \\
Genus g & 1 & 1 & 1 & 1 \\
Surface & $P^{2}$ & $P^{2}$ & $P^{2}$ & $P^{2}$ \\
\hline
\end{tabular}

Table 4: Polygonal embedding for $\Gamma(3 n j)$ graphs of second type. The $\Gamma(9 j)$ graph is separable on 3 edges, 19.

\begin{tabular}{llllll}
\hline Name & $6 j$ & $9 j$ & $12 j$ & $15 j$ & $3 n j$ \\
\hline$|V|=\left|F^{*}\right|$ & 4 & 6 & 8 & 10 & $2 n$ \\
$|E|=\left|E^{*}\right|$ & 6 & 9 & 12 & 15 & $3 n$ \\
$|F|=\left|V^{*}\right|$ & 4 & 5 & 6 & 7 & $n+2$ \\
$\chi$ & 2 & 2 & 2 & 2 & 2 \\
Genus g & 0 & 0 & 0 & 0 & 0 \\
Surface & $S^{2}$ & $S^{2}$ & $S^{2}$ & $S^{2}$ & $S^{2}$ \\
\hline
\end{tabular}
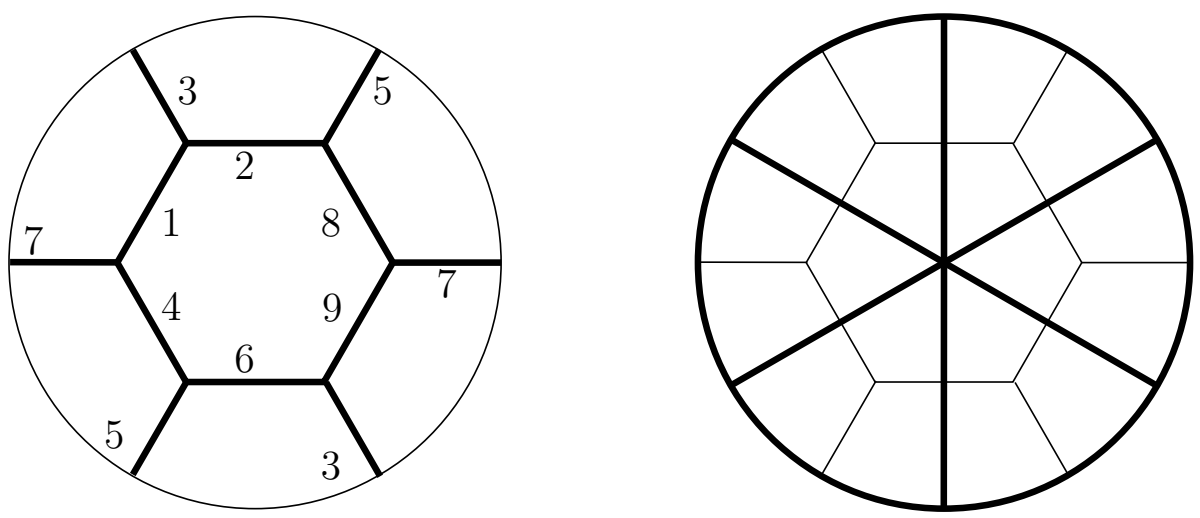

Fig. 4. The graphs of the $9 j$ symbol of the first type embedded into $P^{2}$. A planar drawing is given in [19] Fig. 18.1. Left: 2-skeleton (heavy lines) with 1 hexagon and 3 quadrangles. Right: Dual 2-skeleton (heavy lines) with 6 triangles. 

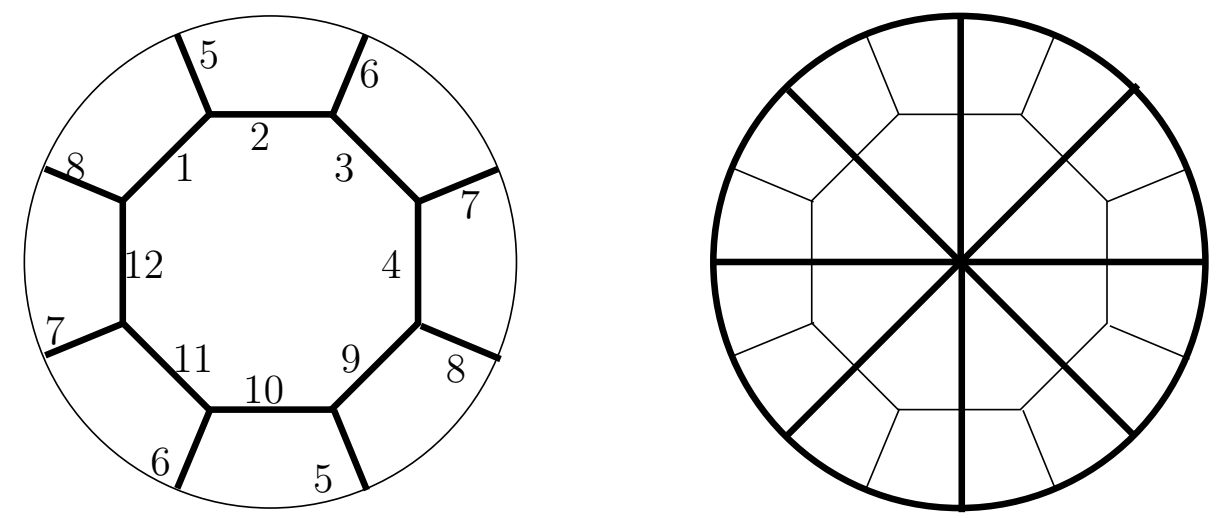

Fig. 5. The graphs of the $12 j$ symbol of the first type embedded into $P^{2}$. Left: $2-$ skeleton (heavy lines) with 1 octagon and 4 quadrangles. A planar drawing is given in 19] Fig. 19.1a. Right: Dual 2-skeleton (heavy lines) with 8 triangles.
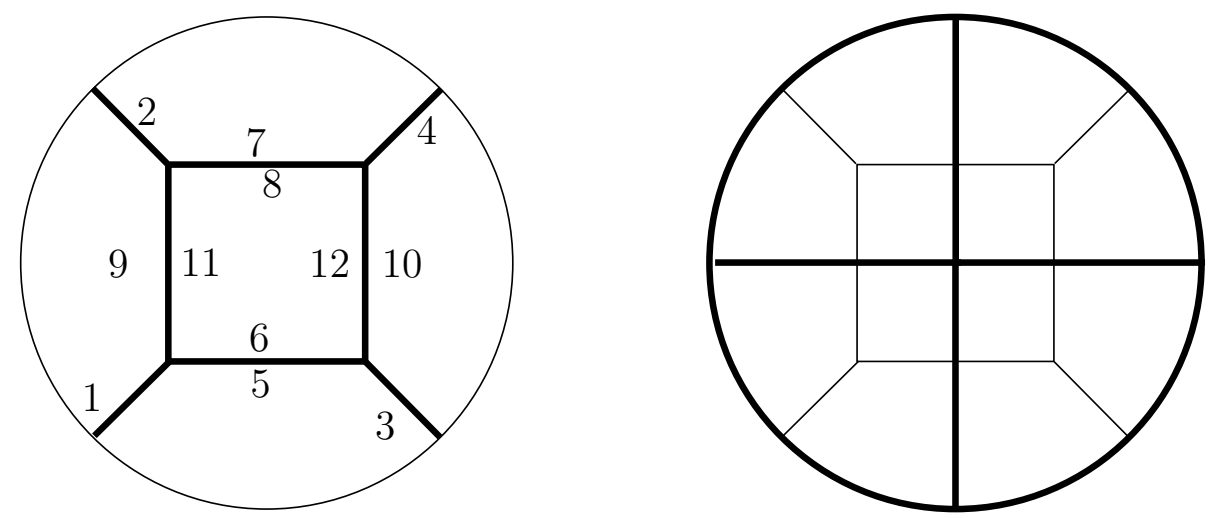

Fig. 6. The graphs of the $12 j$ symbol of the second type embedded into $S^{2}$. Left: cubic 2-skeleton (heavy lines) with 6 quadrangles. The edges $6,8,11,12$ are on top, the edges 5,7, 9, 10 below the equator. A planar drawing is given in [19] Fig. 19.2. Right: Dual octahedral 2-skeleton (heavy lines) with 8 triangles.

For short we use integers $i$ to denote the entries $j_{i}$ of the $3 n j$ symbols. The $9 j$ symbol of the first type with graph shown in Fig. 3 is in the notation of [19] eq. (18.2)

$$
9 j(1):=\left[\begin{array}{lll}
1 & 2 & 3 \\
4 & 5 & 6 \\
7 & 8 & 9
\end{array}\right]
$$

The $12 j$ symbols of the first and second type with graphs shown in Figs. 4 and 5 in the notation of [19] eq. (19.1) and eq.(19.5) respectively are

$$
12 j(1):=\left[\begin{array}{llllllll}
1 & & 2 & & 3 & & 4 & \\
& 5 & & 6 & & 7 & & 8 \\
9 & & 10 & & 11 & & 12 &
\end{array}\right], 12 j(2):=\left[\begin{array}{llll}
1 & 2 & 3 & 4 \\
5 & 6 & 7 & 8 \\
9 & 10 & 11 & 12
\end{array}\right] .
$$


The embeddings of the two families of $3 n j$ graphs into $P^{2}$ and $S^{2}$ are of minimal genus. An important property of the $3 n j$ symbols is their symmetry [19] under certain permutations of the entries $j$. Symmetries keep the numerical value of the corresponding $3 n j$ invariant up to a sign. All of them provide automorphisms of their graphs. A discussion of these symmetries in terms of the surface embeddings is possible but will not be given here.

From the compact surface embeddings of the $3 n j$ graphs one could pass to other embeddings. Any compact surface $\mathcal{S}$ has a simply connected universal covering $\mathcal{U}(\mathcal{S})$. An embedding $\Gamma \subset \mathcal{S}$ may be extended to $\mathcal{U}(\mathcal{S})$. An embedding into $E^{3}$ is obvious for $\Gamma \subset \mathcal{S}=S^{2}$. For the higher embeddings of the projective space $P^{2}$ into $E^{3}$ see [7] pp. 313-329 and into $E^{4}$ pp. 340-342.

\section{$7 \quad$ Dualization of $3 n j$ graphs.}

The concept of dualization, [12] pp. 367-447, applies to general cell complexes $C$ of dimension $d$ and their skeletons or subcomplexes of dimension $m, 0 \leq m \leq d$. For general dimension $d$ of the complex, its dual objects in $C^{*}$ have complementary dimension $(m, d-m) m=0, \ldots, d$. Duality therefore depends on the dimension of the cell complex. For the present cell complexes embedded into surfaces we have $d=2$ and so faces are dual to points and edges dual to edges.

A graph when imbedded into a surface $\mathcal{S}$ extends the graph by its faces to a 2 -skeleton whose 1-skeleton is the graph. For simplicity we shall denote this embedded cell complex again by $\Gamma$. The dual 2-skeleton $\Gamma^{*}$ on the same surface $\mathcal{S}$ may be obtained as follows: As vertices of $\Gamma^{*}$ choose interior points from each face of $\Gamma$. Connect by a dual edge each pair of interior points from pairs of polygons which share an edge. Then the dual edges must close around any vertex of $\Gamma$. Assign a dual face to each such set of closed dual vertices. Clearly the dual graph is embedded into the same surface $\mathcal{S}$. Euler characteristics $\chi$, genus $g$ and orientability are unchanged. The interchange of boundaries follows eq. 4. All dual complexes are triangulations and obey $2\left|E^{*}\right|=3\left|F^{*}\right|$. The new edges of the dual 2-skeletons could carry the same values of $j$ as the old ones.

For the $3 n j$ graphs considered we find for dualization:

3 Prop: The dual 2-skeletons $\Gamma^{*}(3 n j)$ for the first and second type of $\Gamma(3 n j)$ graphs with minimal embedding admit triangulations, non-orientable on the projective space $P^{2}$, and orientable on the sphere $S^{2}$ respectively.

The embeddings and triangulations for the first type of $\Gamma(3 n j)$ graphs into $P^{2}$ should be handled with care: Once the upper and lower hemisphere of the orientable $S^{2}$ are identified on a single non-orientable hemisphere, there is no volume left in between. For higher embeddings of $P^{2}$ compare [7] as quoted above.

The number of vertices, edges and faces and the topological data for the duals are obtained by interchange of the entries for vertices and for faces, see Tables 3 and 4. 
In Figs. 4, 5 and 6 we show examples of polygonal embeddings on the left and their dual triangulations on the right. The generalizations to $n>4$ are straightforward. Non-minimal embeddings with higher genus $g$ could be constructed by the Schwark algorithm with the methods demonstrated in section 5 for the simplex.

Finally we mention an alternative elegant graphical representation of invariants and recoupling coefficients due to Fano and Racah [5] pp. 156-158, see also [14 p. 32. Each triple of coupled vectors in the Fano-Racah-graphs labels 3 points on a straight line. We do not see an easy passage from the Fano-Racah graphs to the topology and dualization in question.

\section{Conclusion.}

The construction of maps due to [3] and [17 allows to obtain all embeddings of spin networks $\Gamma$ into compact surfaces $\mathcal{S}$. The embeddings give insight into a variety of topological data as $\chi, g$, orientability, homotopy and homology, duality and triangulation. For two types of $\Gamma(3 n j)$ networks with general $n$, there are embeddings of minimal genus into the sphere and the projective space. The dual 2-skeletons $\Gamma^{*}(3 n j)$ are triangulations.

We take up the topics of the introduction and draw some conclusion on future applications of spin networks, in particular in the spirit of the initial approach of Regge [15] and Ponzano and Regge [16]. The results reached in this field have been summarized by Regge in [16. In view of the variety of distinct embeddings, topologies and dualizations which we have demonstrated in sections 5 and 6 one could ask for some guidelines on the topology of spin networks.

(1) In our opinion, the embedding of spin networks into surfaces $\mathcal{S}$ and the topological data must be implemented before dualization, triangulation, and before any more advanced geometry and physics of spin networks.

(2) The simplest choice of embeddings would be to admit them only into $\mathcal{S}=S^{2}$. These correspond to convex polyhedra in Euclidean space $E^{3}$, and would allow for standard geometric tools. All $3 n j$ graphs of the second type admit this $g=0$ embedding.

(3) The next possible choice would be embeddings of minimal genus. All $3 n j$ graphs of the first type, beginning with the $9 j$ graph discussed already in [14, admit the embedding into $P^{2}$ with minimal genus $g=1$. Compact embeddings of other characteristics and genus are evident from the embedding of the simplex, section 5 . The projective space $P^{2}$ can be seen as a sphere with the identification of opposite points. But the dual triangulation of $P^{2}$ is not orientable, has no straight-forward interpretation as the boundary of a polytope, and so the geometric view of faces and volumes as suggested in 14 requires further topological qualification.

(4) A more general point of view is suggested by modern cosmology. The study of 
varieties of cosmologies with non-standard topology is in progress [8], and their tests from astronomical evidence are under intense study. Why should discrete models of space not display a similar richness of topology as continuous ones? A restriction to minimal embeddings of graphs may be only a first choice on heuristic grounds. General embeddings of graphs $\Gamma$ into compact surfaces $\mathcal{S}$ with topological data including homotopy and homology, dualization and possible triangulation can illuminate the way towards discrete geometries with richer topology.

(5) Spin networks have been recently applied to quantum gravity [4]. The difference with other approaches discussed in [4] is that they offer new insight into the interpretation of space, a fundamental aspect of which is the correspondence between the combinatorial rules of the networks and the geometrical properties of space. Also a very important consequence of spin networks is that all physical magnitudes are discrete, a property that can be used in the quantization of general relativity [9].

\section{Acknowledgments.}

The authors want to express their gratitude to Professor Bruno Gruber for the invitation to present these ideas in the Symposium SYMMETRIES IN SCIENCES XII. One author (M. L.) gives thanks to the Ministerio de Ciencia y Tecnologia for financial support under grant BFM 2000-0357.

\section{References}

[1] Baez J A, An introduction to spin foam models of back-ground free theory of quantum gravity, in: Geometry and Quantum Physics, eds. H. Gausterer, H. Grosse, L. Pittner, Lecture Notes in Physics 543, Springer, Berlin 2000, 25-95

[2] Biggs N, Algebraic graph theory, Cambridge University Press, Cambridge 1993

[3] Bonnington C P and Little C, The Foundations of Topological Graph Theory, Springer, Berlin 1995

[4] Carlip S, Quantum gravity: a progress report Rep. Progr. Phys. 64 (2001) 885-942 
[5] Fano U and Racah G, Irreducible Tensorial Sets,

Academic Press, New York 1959

[6] Hasslacher B, Perry M J, Spin networks are simplicial quantum gravity, Phys. Lett. B 103 (1981) 21-24

[7] Hilbert D and Cohn-Vossen S, Geometry and the imagination, AMS Chelsea Publishing, Providence 1991

[8] Lachieze-Rey M and Luminet J-P, Cosmic topology,

Physics Reports 254 (1995) 135-214

[9] Loll R, Discrete approaches to quantum gravity in four dimensions, Living Rev. Rel. 1 (1998) 13-46

[10] Lorente M, A causal interpretation of the structure of space and time, in: Foundations of Physics, eds. P. Weingartner and C. Dorn, Hlder-Pichler-Tempsky Verlag, Vienna 1986, pp. 345-368

[11] Lorente M, Quantum processes and the foundation of relational theories of space and time, in: Relativity in general, eds. J. Diaz-Alonso et al., Editions Frontieres, Gif-surYvette Cedex, 1994, 297-302

[12] Munkres J R, Elements of Algebraic Topology,

Addison Wesley, Reading 1984

[13] Penrose R, Angular momentum: an approach to combinatorial space-time, in: Quantum Theory and Beyond, ed. T. Bastin, Cambridge Univ. Press, Cambridge 1971, 151-181

[14] Ponzano G and Regge T, Semiclassical limit of Racah coefficients, in: Spectroscopy and group theoretical methods in Physics, eds. F. Bloch et al., North Holland 1968 
[15] Regge T,

General relativity without coordinates,

Nuoco Cim. 19 (1961) 558-571

[16] Regge T,

Discrete structures in gravity,

J. Math. Phys. 41 (2000) 3964-3984

[17] Schwark M,

The genus of geometric graphs,

Diploma Thesis, Faculty of Mathematics and Computer Science, University of Wrzburg, Germany (2000)

[18] Seifert H and Threlfall W,

Lehrbuch der Topologie,

Reprint Chelsea, New York 1980

[19] Yutsis A P, Levinson I B, and Vanagas V V, Mathematical Apparatus of the Theory of Angular Momentum, Israel Program for Scientific Translations, Jerusalem 1962 